\title{
Conhecimento de gestantes sobre aleitamento materno durante acompanhamento pré-natal em serviços de saúde em Cuiabá
}

\author{
Daniele Merisio Raimundi', Caroline Correa Menezes², Marli Eliane Uecker ${ }^{3}$, \\ Emanuele Batistela Santos ${ }^{4}$, Lorena Barbosa Fonseca ${ }^{5}$
}

\begin{abstract}
RESUMO
O pré-natal é apropriado para o desenvolvimento de estratégias de promoção ao aleitamento materno, assim este estudo objetivou avaliar o conhecimento de gestantes sobre o tema durante este período. Realizou-se um estudo exploratório, descritivo, transversal, de abordagem quantitativa, com 60 gestantes em espera para atendimento pré-natal em serviços de saúde de Cuiabá-MT. Os dados foram coletados entre julho e dezembro de 2014 por meio de questionário semiestruturado abordando o conhecimento sobre aleitamento materno. Do total de participantes, $41,67 \%$ referiram já ter recebido orientação sobre aleitamento materno. Entre as vantagens do leite materno destacaram-se sua qualidade nutricional e imunológica. O período adequado de aleitamento materno exclusivo e o não estabelecimento de horários fixos para amamentar foram conhecidos pela maioria, o mesmo não ocorrendo em relação às complicações. Este estudo soma-se à literatura, demonstrando aspectos menos conhecidos sobre 0 aleitamento materno e oferece subsídios para a prática profissional baseada na aprendizagem significativa.
\end{abstract}

Descritores: Amamentação; Assistência Pré-natal; Promoção da Saúde.

\section{Breastfeeding knowledge among pregnant women during prenatal care in health units in Cuiabá}

\begin{abstract}
Prenatal is suitable for the development of strategies to promote breastfeeding, so this study aimed to evaluate the knowledge of pregnant women on the subject during this period. We performed an exploratory, descriptive, cross-sectional study with a quantitative approach, with 60 pregnant women waiting for prenatal care in health care Cuiaba-MT. Data were collected between July and December 2014 through semi-structured questionnaire addressing knowledge about breastfeeding. Of total participants, $41.67 \%$ said they have already received guidance on breastfeeding. Among the advantages of breast milk stood out its nutritional and immunological quality. The appropriate duration of exclusive breastfeeding and not the establishment of fixed breastfeeding schedules have been known by the majority, which did not occur in relation to complications. This study adds to the literature by demonstrating the lesser known aspects of breastfeeding and provides grants for professional practice based on meaningful learning.
\end{abstract}

Descriptors: Breast Feeding; Prenatal Care; Health Promotion.

${ }^{1}$ Graduada em Enfermagem pela Universidade Federal de Mato Grosso (UFMT), Cuiabá, MT, Brasil.

${ }^{2}$ Graduanda em Nutrição na Universidade Federal de Mato Grosso (UFMT), Cuiabá, MT, Brasil.

${ }^{3}$ Mestre em Saúde Coletiva pela Universidade Federal de Mato Grosso (UFMT), Cuiabá, MT, Brasil.

${ }^{4}$ Mestre em Biociências pela Universidade Federal de Mato Grosso (UFMT), Cuiabá, MT, Brasil.

${ }^{5}$ Mestre em Ciência da Nutrição pela Universidade Federal de Viçosa (UFV), Viçosa, MG, Brasil. 


\section{Introdução}

O aleitamento materno exclusivo (AME) nos seis primeiros meses de vida e sua continuidade pelo menos até os dois anos de idade, acompanhado de alimentos complementares, tem fundamental importância na redução dos riscos de morbimortalidade infantil ${ }^{1}$. Ainda assim, dados do Ministério da Saúde ${ }^{2}$ apontaram que Cuiabá/MT apresentou, no ano de 2009, a menor prevalência de AME em dias amamentados entre as capitais brasileiras (0,742 dias). Mais que 50\% das crianças não se encontrava em AME no primeiro dia de vida, classificando a capital mato-grossense como a primeira em desmame precoce. Este pode ser resultado de uma série de fatores socioculturais, entre eles a inserção da mulher no mercado de trabalho, a ampla divulgação na mídia e entre alguns profissionais de saúde de leites industrializados, mitos sobre o aleitamento materno (AM), intercorrências mamárias, como ingurgitamento e fissuras, além do desconhecimento da mãe em relação ao tema ${ }^{3,4}$.

Diante disso, o desenvolvimento de estratégias de cunho educativo cuja abordagem seja voltada para as vantagens do aleitamento materno e para o manejo adequado das intercorrências que possam surgir durante sua prática é urgente e necessário.

Muitas vezes, o início da amamentação é um processo difícil para a mãe. Desta forma, o conhecimento adquirido por meio de ações de educação em saúde pode facilitar a prática do $\mathrm{AM}^{4}$

A literatura aponta que o período em que as estratégias são desenvolvidas influencia no grau de adesão às recomendações, de maneira que o pré-natal parece ser o momento mais apropriado para a aplicação de tais ações pois, diferente do pós-parto, a atenção da mãe não está totalmente voltada aos cuidados com o recém-nascido, e encontrase, portanto, mais aberta à novas informações ${ }^{5-7}$.Desta forma, as orientações passadas durante a atenção pré-natal contribuem significativamente para sensibilizar a mulher quanto ao aleitamento materno $0^{6,8}$, considerando que para a maioria delas, a decisão de amamentar acontece muito antes do parto.

Merece salientar, no entanto, que as estratégias educativas devem ir além da orientação acerca da importância do AM, uma vez que, embora as mães conheçam suas vantagens para a criança, apresentam dificuldades com questões simples, tais como a técnica de sucção e os cuidados com as mamas para a lactação, por desconhecimento de tais aspectos, conforme destaca Freitas et al. ${ }^{4}$. Além disso, a informação por si só muitas vezes não é suficiente para a prática do AM. Estudo de Santana et al. ${ }^{9}$ demonstrou que embora as mães tivessem conhecimento sobre o AM, este nem sempre foi aplicado.

Isso revela a importância da assistência humanizada, que considere o suporte adequado à mãe, no contexto do desenvolvimento das estratégias de promoção ao AM. Estudos mostram que a maior parte das dificuldades quanto à prática da amamentação é consequência de falhas na assistência à gestante durante o pré-natal, destacando, portanto, a importância da comunicação, do vínculo e do apoio dos profissionais de saúde para a eficácia das ações 5,10 .

Neste sentido, o grupo tutorial do Programa de Educação pelo Trabalho para a Saúde (PET-Saúde)/Rede Cegonha, da Universidade Federal de Mato Grosso (UFMT), através da metodologia da problematização, planejou e executou um programa de apoio, promoção e incentivo ao aleitamento materno durante o pré-natal, com duração aproximada de um ano. As atividades deste programa caracterizaram-se por ações de cunho educativo, baseadas em metodologias ativas, desenvolvidas na sala de espera de um hospital universitário do município de Cuiabá-MT, que possui um Banco de Leite Humano e em unidades básicas de saúde (UBS) que referenciavam as gestantes àquele hospital.

Diante do exposto, estudos que investiguem o conhecimento acerca do AM são importantes para subsidiar a implantação de estratégias adequadas de promoção, incentivo e apoio ao AM. O presente estudo se desenvolveu a partir da questão norteadora "Qual o conhecimento de gestantes sobre a prática do aleitamento materno?". Deste modo, objetivou-se avaliar o conhecimento das gestantes sobre o aleitamento materno durante 0 acompanhamento pré-natal.

\section{Metodologia}

Estudo do tipo exploratório, descritivo, transversal, de abordagem quantitativa realizado em um hospital universitário, cenário de prática das atividades do PET-Saúde/Rede Cegonha da UFMT e em 15 unidades básicas de saúde (UBS) do município de Cuiabá/MT. Estas unidades foram escolhidas por se tratarem de serviços que referenciavam as gestantes para o hospital.

Os participantes do estudo foram gestantes que se encaminharam ao hospital ou UBS para acompanhamento prénatal. Nas UBS, um convite individualizado foi enviado à gestante via Agente Comunitário de Saúde, para participação em uma atividade educativa sobre aleitamento materno realizada pelo grupo tutorial do PET-Saúde/Rede Cegonhal UFMT. Em todas as UBS, estas atividades foram agendadas para os dias reservados às consultas de pré-natal. Nas 
salas de espera, antes do início das atividades educativas, as gestantes foram convidadas a participar da pesquisa. Foram excluídas gestantes menores de idade sem a presença de um responsável. Aquelas que não completaram a entrevista também foram excluídas do estudo.

A coleta de dados foi realizada entre os meses de julho e dezembro de 2014, por meio de questionário semiestruturado, elaborado pelos próprios pesquisadores a partir dar recomendações sobre aleitamento materno do Ministério da Saúde e aplicado pelos monitores do PET-Saúde. O questionário era dividido em duas partes, sendo a primeira composta por dados socioeconômicos e obstétricos, como situação conjugal, escolaridade, profissão, número de gestações anteriores, história prévia de amamentação e realização de pré-natal, e a segunda por variáveis relativas ao conhecimento sobre aleitamento materno, como vantagens, duração recomendada, livre demanda, mitos, entre outros.

Para apuração e análise dos dados utilizou-se o Software Epi Info versão 7. Para verificar a existência de associação entre as variáveis categóricas foi utilizado o teste de $x^{2}$. Para comparação entre médias, foi utilizado o teste t de Student para variáveis paramétricas e o teste de Mann-Whitney para as não paramétricas. Foi adotado como nível de significância estatística um valor de $p<0,05$ para todas as análises.

Todas as gestantes receberam esclarecimento sobre sua participação na pesquisa e foram orientadas a assinarem, previamente ao preenchimento do questionário, um Termo de Consentimento Livre e Esclarecido (TCLE), elaborado de acordo com a Resolução 466/2012. Este estudo foi aprovado pelo Comitê de Ética do Hospital Universitário Júlio Müller sob parecer $n^{0} 735.643$, de 31 de julho de 2014.

\section{Resultados e discussão}

Durante o período da coleta de dados, um total de 60 gestantes (idade gestacional mediana de 28 semanas; p25=16,5-p75=34) foram entrevistadas na sala de espera do hospital e UBS. A Tabela 1 apresenta o resultado da análise das características sociodemográficas.

Como pode-se observar, a maioria das gestantes residia no município de Cuiabá/MT. A análise das características sociodemográficas permitiu identificar que a maior parte era solteira, se autodeclarava parda, possuía ensino médio completo ou mais anos de estudo e tinha carteira assinada. A média de idade foi de 27 anos, tendo esta variado entre 13 a 43 anos.

Tabela 1 - Perfil sociodemográfico de gestantes atendidas em pré-natal em serviços públicos de Cuiabá-MT. Cuiabá, 2014.

\begin{tabular}{c|c|c}
\hline Variável & $\mathrm{n}$ & $\%$ \\
\hline Residência & & 82,76 \\
\hline Cuiabá & 48 & 8,62 \\
\hline Várzea Grande & 05 & 8,62 \\
\hline Outros & 05 & \\
\hline Estado civil & & 43,33 \\
\hline Casada & 26 & 55,00 \\
\hline Solteira & 33 & 1,67 \\
\hline Viúva & 01 & 11,67 \\
\hline Cor/Raça & & 50,00 \\
\hline Branca & 07 & 35,00 \\
\hline Parda & 30 & 3,33 \\
\hline Negra & 21 & 0,00 \\
\hline Amarela & 02 & \\
\hline Indígena & 00 & Continua...
\end{tabular}




\begin{tabular}{c|c|c}
\hline Variável & $\mathbf{n}$ & $\%$ \\
\hline Escolaridade & & 6,67 \\
\hline Ensino fundamental incompleto & 04 & 10,00 \\
\hline Ensino fundamental completo & 06 & 20,00 \\
\hline Ensino médio incompleto & 12 & 40,00 \\
\hline Ensino médio completo & 24 & 10,00 \\
\hline Ensino superior incompleto & 06 & 13,33 \\
\hline Ensino superior completo & 08 & \\
\hline Carteira Assinada & & 52,27 \\
\hline Sim & 23 & 47,73 \\
\hline Não & 21 & \\
\hline
\end{tabular}

Estudos mostram que alguns fatores como a escolaridade e a idade materna podem interferir na decisão da mãe em amamentar ou não seu filho. Em geral, a maior escolaridade e a idade superior a 20 anos aumentam as chances de a mãe amamentar. Acredita-se que isso se deve ao fato de estas características se associarem à maior estabilidade e segurança da mãe ${ }^{11-13}$. Entretanto, neste estudo, não se observou associação entre a idade e a escolaridade e ter ou não amamentado ( $x^{2}=3,39, p=0,06$ e $x^{2}=1,30, p=0,25$, respectivamente).

Com relação aos antecedentes obstétricos, observou-se uma média de 2,30 gestações $( \pm 1,29)$ e uma frequência de 15 abortos. 0 parto normal foi o mais frequente $(n=25 ; 71,42 \%)$. A média de idade na primeira gestação foi de 21,66 anos $( \pm 6,72)$. Das gestantes multíparas ( $n=35), 94,12 \%(n=33)$ amamentaram anteriormente. A mediana de duração de aleitamento materno do primeiro filho foi de 13,50 meses, e do segundo filho de nove meses (média de 13,8 e 11,8 meses, respectivamente; $p=0,7$ ).

Observou-se que a mediana da duração de amamentação encontrada neste estudo, para o primeiro filho, foi menor do que a recomendação do Ministério da Saúde, que é, de pelo menos 24 meses, entretanto, foi maior do que a mediana nacional e a do município de Cuiabá, que eram, no ano de 2009, 11,40 meses e 12,99 meses, respectivamente ${ }^{1}$.

Orientações sobre aleitamento materno são fundamentais no pré-natal, entretanto, apenas 25 gestantes $(41,67 \%)$ referiram ter recebido algum tipo de orientação anteriormente à entrevista. O local de recebimento destas orientações variou entre as gestantes, sendo que $42,31 \%(n=11)$ receberam no pré-natal anterior, $26,92 \%(n=7)$ na maternidade, $19,23 \%$ $(n=5)$ no pré-natal atual e $11,54 \%(n=3)$ referiram ter recebido orientações em outro momento. Não se observou associação entre o município de residência da gestante e o recebimento de orientações $\left(X^{2}=1,05 ; p=0,5\right)$. Também não se observou associação entre ter recebido orientação sobre aleitamento materno e ter ou não amamentado seus filhos anteriores $\left(X^{2}=\right.$ $3,38 ; p=0,18)$, refletindo a ampla gama de fatores que se associam à decisão da mãe em amamentar seu filho. Muitas vezes, o conhecimento da importância da amamentação não é suficiente para sua realização, por outro lado, estudos apontam para a importância do adequado conhecimento sobre amamentação, uma vez que uma das razões mais frequentemente associadas à prática do AM é justamente o conhecimento sobre os benefícios que o leite materno proporciona à criança ${ }^{14,15}$.

Em estudo realizado na capital do Rio de Janeiro com 71 mulheres, os autores observaram, assim como neste estudo, que um pequeno percentual de gestantes recebeu orientações sobre aleitamento materno durante o pré-natal. Considerando a importância deste período para o sucesso da amamentação, acredita-se que os profissionais de saúde devem ser sensibilizados quanto ao impacto que esta prática pode exercer ${ }^{15}$.

$\mathrm{Na}$ sala de espera, anteriormente ao desenvolvimento das atividades de educação em saúde e de promoção do aleitamento materno, as gestantes $(n=55)$ responderam a um questionário contendo informações sobre o tema para a verificação do conhecimento das mesmas.

Sobre as vantagens do aleitamento materno, as respostas mais frequentes foram "é o alimento mais saudável", "previne doenças", "é como uma vacina", conforme observado no Gráfico 1. Estudo realizado com mães de lactentes cadastradas no Programa de Saúde da Família do município de Teixeiras (MG) revelou que a maioria delas (39\%) acreditava que a maior vantagem do leite materno associava-se com a imunização da criança ${ }^{16}$. Sobre 0 período adequado para 0 aleitamento materno exclusivo, pode-se observar no Gráfico 2 que a maioria respondeu corretamente ( $n=33 ; 61,11 \%$ ). Sobre estipular horário para amamentação, a maior parte das gestantes $(n=31 ; 57,41 \%)$ afirmou não ser necessário, mas considera-se alto o número de mães que acreditavam que o estabelecimento de horários fixos era necessário. 
Nota-se que quando questionadas sobre as vantagens do AM a maioria mencionou benefícios para a criança, não levando em conta a vantagem para mulher que amamenta. Segundo Rea ${ }^{17}$ os benefícios da amamentação à saúde da mulher apesar de serem muito relevantes, ainda são desconhecidos pelas mulheres, e raramente são divulgados pelos profissionais e serviços de saúde.

Quanto ao horário fixo para amamentar, estudo aponta que as mães deveriam ser orientadas quanto ao fato de que crianças menores não têm hábito de praticar alimentações regulares e que estabelecer horários fixos para amamentar pode diminuir a produção de leite materno, e consequentemente aumentar a fome e a irritabilidade do bebê ${ }^{14}$.

Quando questionadas sobre a existência de alguma situação que impossibilita a mãe de amamentar, as gestantes levantaram algumas situações, como mães com doenças transmissíveis, mães que precisam tomar medicamentos prejudiciais ao bebê, usuárias de drogas e, que não possuem leite.

Em estudo realizado da cidade de Passo Fundo/RS, um dos principais fatores relacionados ao desmame precoce foram as dificuldades da amamentação nos primeiros dias de vida da criança, e a crença de não possuir leite ou ter leite fraco. Evidencia-se, portanto, a necessidade do apoio profissional ${ }^{18}$.

Neste estudo, as gestantes foram questionadas também acerca do conhecimento sobre mitos, como o leite fraco, sobre os benefícios do aleitamento materno e sobre modos de aumentar a produção de leite. A maioria ( $n=39 ; 73,58 \%)$ das gestantes referiu que não existe leite fraco, que as crianças amamentadas pegam menos doenças ( $n=46 ; 88,46 \%$ ) e que para aumentar a produção de leite a mãe pode aumentar a ingestão de líquidos ( $n=16 ; 30,19 \%$ ), ou apontaram outra coisa a se fazer.

Sobre os problemas mais comumente observados durante a amamentação, como rachaduras no bico do seio, ingurgitamento mamário e mastite, as gestantes foram questionadas sobre formas de prevenção e tratamento. Em relação à prevenção de fissuras, ingurgitamento mamário e mastite a maioria das gestantes não soube responder ( $n=18 ; 33,96 \%, n=21$; $39,62 \%$ e n=40; 76,92\% respectivamente), e 28,30\% ( $n=15)$ referiram "passar pomadas e cremes" como um método de prevenção para fissuras. Já com relação ao tratamento destes problemas, para fissuras, a maioria ( $n=23 ; 44,23 \%)$ respondeu "passar pomadas e cremes". Para o ingurgitamento e mastite, a maioria disse não saber ( $n=27 ; 51,92 \%$ e n=40; 76,92\%, respectivamente).

Destaca-se que as mães muitas vezes recebem informações como a importância do aleitamento materno, posição e pega, prevenção e tratamento de problemas mamários. Porém, ao se depararem com essas situações no retorno para casa, se tornam susceptíveis ao desmame precoce devido à inexperiência e até mesmo a impaciência ${ }^{18}$. Neste caso, seria importante que essas atividades fossem realizadas juntamente com o parceiro da gestante, ou alguém com maior proximidade da mesma, para que deste modo, possa auxiliá-la e apoiá-la no momento pós-parto.

Tendo em vista o exposto neste trabalho, observa-se a relevância de inserção de atividade de promoção e apoio ao AM em hospitais e UBS no período pré e pós-natal, pois, resultará em melhor qualidade de vida e saúde para a nutriz e recém-nascido, além de menores custos ao sistema de saúde do país.

\section{Considerações Finais}

No presente estudo percebeu-se que quando questionadas sobre a importância do aleitamento materno, a maioria das gestantes demonstrou ter conhecimento sobre as principais vantagens de sua prática, já quando se tratando dos problemas relacionados ao AM e o tratamento e prevenção desses agravos a maior parte das entrevistadas não soube responder corretamente.

A literatura aponta uma série de benefícios proporcionados pelo aleitamento materno para saúde da mãe e da criança, portanto, é de suma importância que os serviços de saúde estabeleçam estratégias de promoção, apoio e incentivo ao aleitamento materno. Sugere-se ainda que o acompanhamento sobre o aleitamento materno seja realizado também no pós-parto, pois é o momento em que as mulheres estão expostas as dificuldades no processo de estabelecimento do aleitamento materno.

Este estudo soma-se à literatura, demonstrando os aspectos pouco conhecidos sobre o aleitamento materno entre as gestantes, de maneira a oferecer subsídios para o estabelecimento de práticas profissionais que considerem a aprendizagem significativa.

Destacam-se como limitações do estudo, o fato dos resultados refletirem o conhecimento relatado por gestantes atendidas em um conjunto restrito de serviços de saúde e a ausência de investigação sobre a pretensão das gestantes de amamentar. Cabe, portanto, o desenvolvimento de novos estudos à nível regional, considerando a gama de fatores determinantes que influenciam a prática do aleitamento materno, bem como o desenvolvimento de programas de promoção do aleitamento materno, focando especialmente no suporte à sua prática. 


\section{Referências Bibliográficas}

1. Brasil, Ministério da Saúde. Secretaria de Assistência à Saúde. Manual de promoção do aleitamento materno. Brasília: Ministério da Saúde, 2009.

2. Brasil. Ministério da Saúde. Secretaria de Assistência à Saúde. II Pesquisa de Prevalência de Aleitamento Materno nas Capitais Brasileiras e Distrito Federal. Brasília: Ministério da Saúde, 2009.

3. Prazeres MAF, Rank RCIC, Rank MS, Rezende JEV . "Cantinho da mamãe." Educação sobre aleitamento materno por meio de vídeos para puérperas no Hospital: relato de caso . Revista Amazônia Science \& Health. 2015;3(1):27-32.

4. FREITAS, G.L.; JOVENTINO, E.S.; AQUINO, P.S.; PINHEIRO, A.K.B.; XIMENES, L.B. Avaliação do conhecimento de gestantes acerca da amamentação

Rev. Min. Enferm. 2008;12(4): 461-468.

5. Demito MO, Silva TC, Páschoa ARZ, Mathias TAF, Bercini LO. Orientações sobre amamentação na assistência prénatal: uma revisão integrativa. Rev. Rene. 2010 11(Número Especial): 223-229.

6. Nascimento VC, Oliveira MIC, Alves VH, Silva KS. Associação entre as orientações pré-natais em aleitamento materno e a satisfação com o apoio para amamentar. Rev. Bras. Saúde Mater. Infant., Recife.2013 Abr; 13(2): 147-159.

7. Takemoto AY, Santos AL, Okubo P, Bercini LO, Marcon SS. Preparo e apoio à mãe adolescente para a prática de amamentação. Cienc Cuid Saude. 2011 Jul/Set; 10(3): 444-451.

8. Nascimento VC. Orientações sobre aleitamento materno prestadas no pré-natal de hospitais do SUS e sua associação com a satisfação das gestantes quanto ao apoio recebido para amamentar [dissertação]. Niterói: Universidade Federal Fluminense; 2012. 9. Santana, JM; Brito, SM; Santos, DB. Amamentação: conhecimento e prática de

Gestantes. O Mundo da Saúde, São Paulo-2013;37(3):259-267.

10. Souza MJN, Barnabé AS, Oliveira RS, Ferraz RRN. A importância da orientação à gestante sobre amamentação: fator para diminuição dos processos dolorosos mamários. ComScientiae Saúde. 2009 8(2): 245-249.

11. França GVA, Brunken GS, Silva SM, Escuder MM, Venancio SI. Determinantes da amamentação no primeiro ano de vida em Cuiabá, Mato Grosso. Rev. Saúde Pública. 2007 Out; 41( 5 ): 711-718.

12. Takushi SAM, Tanaka ACA, Gallo PR, Machado MAMP. Motivação de gestantes para o aleitamento materno. Rev Nutr. 2008 Set/Out; 21(5): 491-502.

13. BrittoLF. Orientação e incentivo ao aleitamento materno na assistência pré-natal e puerperal: uma revisão de literatura. Rev Saúde Públ. 2013 6(1): Jan/Mar; 66-80.

14. Giugliani ERJ, Rocha VLL, Neves JM, Polanczy CA, Seffrin CF, Susin LO. Conhecimentos maternos em amamentação e fatores associados. Jornal de Pediatria. 1995; 71(2): 77-81.

15.Sandre-Pereira G, Colares LGT, Carmo MGT, Soares EA. Conhecimentos maternos sobre amamentação entre puérperas inscritas em programa de pré-natal. Cad. Saúde Pública. 2000 June; 16(2): 457-466

16. Azeredo CM, Maia TM, Rosa TCA, Silva FF, Cecon PR, Cotta RMM. Percepção de mães e profissionais de saúde sobre o aleitamento materno: encontros e desencontros. Rev Paul Pediatr. 2008;26(4):336-44.

17. Rea MF. Os benefícios da amamentação para a saúde da mulher. Jornal de Pediatria. 2004; 80(5 Supl):S142-S146.

18. Giugliani ERJ, Lamounier JA. Aleitamento materno: uma contribuição científica para a prática do profissional de saúde Jornal de Pediatria. 2004; 80(5 Supl):S117-S118. 


\section{Daniele Merisio Raimundi}

Endereço para correspondência - Rua: Av. Fernando Corrêa da Costa, $n^{\circ} 2367$, Bairro: Boa Esperança, CEP: 78060-900, Cuiabá, MT, Brasil.

E-mail: dani.raimundi@gmail.com

Lattes: http://lattes.cnpq.br/4644724014500672

Caroline Correa Menezes - carolinecorreamenezes@gmail.com

Marli Eliane Uecker - marlieliane@yahoo.com.br

Emanuele Batistela Santos - emanuelebatistela.ufmt@gmail.com

Lorena Barbosa Fonseca - lorenanutricao@gmail.com

\section{Enviado em 15 de maio de 2015.}

Aceito em 14 de outubro de 2015. 
